\title{
ON THE SPECTRA AND PSEUDOSPECTRA OF A CLASS OF NON-SELF-ADJOINT RANDOM MATRICES AND OPERATORS
}

\author{
Simon N. Chandler-Wilde, Ratchanikorn Chonchaiya \\ AND MARKO LINDNER
}

Abstract. In this paper we develop and apply methods for the spectral analysis of non-selfadjoint tridiagonal infinite and finite random matrices, and for the spectral analysis of analogous deterministic matrices which are pseudo-ergodic in the sense of E. B. Davies (Commun. Math. Phys. 216 (2001), 687-704). As a major application to illustrate our methods we focus on the "hopping sign model" introduced by J. Feinberg and A. Zee (Phys. Rev. E 59 (1999), 6433-6443), in which the main objects of study are random tridiagonal matrices which have zeros on the main diagonal and random \pm 1 's as the other entries. We explore the relationship between spectral sets in the finite and infinite matrix cases, and between the semi-infinite and bi-infinite matrix cases, for example showing that the numerical range and $p$-norm $\varepsilon$ pseudospectra $(\varepsilon>0, p \in[1, \infty])$ of the random finite matrices converge almost surely to their infinite matrix counterparts, and that the finite matrix spectra are contained in the infinite matrix spectrum $\Sigma$. We also propose a sequence of inclusion sets for $\Sigma$ which we show is convergent to $\Sigma$, with the $n$th element of the sequence computable by calculating smallest singular values of (large numbers of) $n \times n$ matrices. We propose similar convergent approximations for the 2-norm $\varepsilon$-pseudospectra of the infinite random matrices, these approximations sandwiching the infinite matrix pseudospectra from above and below.

Mathematics subject classification (2010): Primary 47B80; Secondary 47A10, 47B36.

Keywords and phrases: random matrix, spectral theory, Jacobi matrix, operators on $\ell^{p}$.

\section{REFERENCES}

[1] A. BöTtChER, Pseudospectra and singular values of large convolution operators, J. Integral Equations Appl. 6 (1994), 267-301.

[2] A. BÖTTCHER, M. EMBREE AND M. LindneR, Spectral approximation of banded Laurent matrices with localized random perturbations, Integral Equations and Operator Theory 42 (2002), 142-165.

[3] A. Böttcher, M. Embree And V. I. Sokolov, Infinite Toeplitz and Laurent matrices with localized impurities, Linear Algebra Appl. 343/344 (2002), 101-118.

[4] A. Böttcher, M. Embree And V. I. Sokolov, On large Toeplitz band matrices with an uncertain block, Linear Algebra Appl. 366 (2003), 87-97.

[5] A. BötTCher, M. EMbReE AND V. I. SOKOLOV, The spectra of large Toeplitz band matrices with a randomly perturbed entry, Mathematics of Computation 72 (2003), 1329-1348.

[6] A. Böttcher And S. M. GRudsky, Spectral Properties of Banded Toeplitz Matrices, SIAM, Philadelphia 2005.

[7] A. BötTCher, S. M. GRUdSky And B. Silbermann, Norms of inverses, spectra, and pseudospectra of large truncated Wiener-Hopf operators and Toeplitz matrices, New York J. Math. 3 (1997), 1-31.

[8] M. CAPInski And E. P. Kopp, Measure, Integral and Probability, Springer Verlag 2004.

[9] R. CARmona And J. Lacroix, Spectral Theory of Random Schrödinger Operators, Birkhäuser, Boston 1990.

[10] S. N. Chandler-Wilde, R. Chonchaiya And M. Lindner, Eigenvalue problem meets Sierpinski triangle: computing the spectrum of a non-self-adjoint random operator, Operators and Matrices 5 (2011), 633-648. 
[11] S. N. Chandler-Wilde, R. Chonchaiya And M. Lindner, Upper bounds on the spectra and pseudospectra of Jacobi and related operators, in preparation.

[12] S. N. ChandLeR-WILdE AND E. B. Davies, Spectrum of a Feinberg-Zee random hopping matrix, Journal of Spectral Theory, Preprint at arXiv:1110.0792, to appear.

[13] S. N. Chandler-WILde AND M. Lindner, Sufficiency of Favard's condition for a class of banddominated operators on the axis, J. Funct. Anal. 254 (2008), 1146-1159.

[14] S. N. Chandler-Wilde And M. Lindner, Limit Operators, Collective Compactness, and the Spectral Theory of Infinite Matrices, Memoirs AMS 210, 989 (2011).

[15] R. ChonchaiYa, Computing the Spectra and Pseudospectra of Non-Self-Adjoint Random Operators Arising in Mathematical Physics, PhD Thesis, University of Reading, UK, 2010.

[16] G. M. Cicuta, M. Contedini ANd L. Molinari, Non-Hermitian tridiagonal randommatrices and returns to the origin of a random walk, J. Stat. Phys. 98 (2000), 685-699.

[17] G. M. Cicuta, M. CONTEdini AND L. Molinari, Enumeration of simple random walks and tridiagonal matrices, J. Phys. A Math. Gen. 35 (2002), 1125-1146.

[18] E. B. Davies, Spectral properties of non-self-adjoint matrices and operators, Proc. Royal Soc. A. 457 (2001), 191-206.

[19] E. B. DaVIES, Spectral theory of pseudo-ergodic operators, Commun. Math. Phys. 216 (2001), 687704.

[20] E. B. Davies, Linear Operators and their Spectra, Cambridge University Press, 2007.

[21] J. Feinberg And A. ZeE, Non-Hermitean Localization and De-Localization, Phys. Rev. E 59 (1999), 6433-6443.

[22] J. Feinberg And A. ZeE, Spectral Curves of Non-Hermitean Hamiltonians, Nucl. Phys. B 552 (1999), 599-623.

[23] J. GlobeVniK, Norm-constant analytic functions and equivalent norms, Illinois J. Math. 20 (1976), 503-506.

[24] I. GOLDSHEID AND B. KHORUZHENKO, Eigenvalue curves of asymmetric tridiagonal random matrices, Electronic Journal of Probability 5 (2000), 1-28.

[25] R. Hagen, S. Roch and B. Silbermann, $C^{*}$-Algebras and Numerical Analysis, Marcel Dekker, New York, 2001.

[26] N. Hatano and D. R. Nelson, Vortex Pinning and Non-Hermitian Quantum Mechanics, Phys. Rev. B 56 (1997), 8651-8673.

[27] D.E. Holz, H. ORland And A. ZeE, On the remarkable spectrum of a non-Hermitian random matrix model, J. Phys. A Math. Gen. 36 (2003), 3385-3400.

[28] K. JöRgens, Linear Integral Operators, Pitman, Boston, 1982.

[29] T. Kato, Perturbation Theory for Linear Operators, 2nd edition, Springer, New York, 1980.

[30] B. V. LANGE AND V. S. RabinOVICH, On the Noether property of multidimensional discrete convolutions, Mat.Zametki 37 (1985), 407-421 (Russian; English transl. Math. Notes 37 (1985), 228-237).

[31] M. Lindner, Infinite Matrices and their Finite Sections: An Introduction to the Limit Operator Method, Frontiers in Mathematics, Birkhäuser 2006.

[32] M. LindneR, Fredholm Theory and Stable Approximation of Band Operators and Generalisations, Habilitation thesis, TU Chemnitz, Germany, 2009.

[33] M. Lindner, Fredholmness and index of operators in the Wiener algebra are independent of the underlying space, Operators and Matrices 2 (2008), 297-306.

[34] M. LindnER, A note on the spectrum of bi-infinite bi-diagonal random matrices, Journal of Analysis and Applications 7 (2009), 269-278.

[35] M. Lindner AND S. Roch, Finite sections of random Jacobi operators, SIAM J. Numer. Anal., Preprint at arXiv:1011.0907v1, to appear.

[36] C. MARTÍNEZ ADAME, On the spectral behaviour of a non-self-adjoint operator with complex potential, Math. Phys. Anal. Geom. 10 (2007), 81-95.

[37] D. R. Nelson And N. M. Shnerb, Non-Hermitian localization and population biology, Phys. Rev. E 58 (1998), 1383-1403.

[38] L. A. Pastur And A. L. Figotin, Spectra of Random and Almost-Periodic Operators, Springer, Berlin 1992.

[39] V. S. Rabinovich, S. Roch And B. Silbermann, Fredholm theory and finite section method for band-dominated operators, Integral Equations Operator Theory 30 (1998), 452-495. 
[40] V. S. Rabinovich, S. Roch And B. Silbermann, Limit Operators and Their Applications in Operator Theory, Birkhäuser, 2004.

[41] L. REICHEL AND L. N. TREFETHEN, Eigenvalues and pseudo-eigenvalues of Toeplitz matrices, Linear Algebra and Appl. 162 (1992), 153-185.

[42] S. Roch, Numerical ranges of large Toeplitz matrices, Lin. Alg. Appl. 282 (1998), 185-198.

[43] E. SHARGORODSKY, On the level sets of the resolvent norm of a linear operator, Bull. London Math. Soc. 40 (2008), 493-504.

[44] E. M. Stein And G. WeIss, Introduction to Fourier analysis on Euclidean spaces, Princeton University Press, Princeton, 1971.

[45] L. N. Trefethen, M. Contedini And M. Embree, Spectra, pseudospectra, and localization for random bidiagonal matrices, Comm. Pure Appl. Math. 54 (2001), 595-623.

[46] L. N. TREFETHEN AND M. EMBREE, Spectra and pseudospectra: the behavior of nonnormal matrices and operators, Princeton University Press, Princeton, NJ, 2005. 